\title{
Advances in Flash Thermography for NDT and Materials Characterization
}

\author{
Steven M. Shepard, Thermal Wave Imaging, Inc., Ferndale, MI USA
}

\begin{abstract}
\end{abstract}
In the past decade, active thermography has emerged from the research laboratory and evolved to become a mainstream NDT technique for numerous applications in the aerospace, power generation and automotive industries. Evidence of this trend can be found with the growing number of companies that use thermography as a first-line, standalone NDT technique, rather than as a qualitative adjunct to a primary method, or in the recent publication of ATSM- E2582- 07, which represents a consensus of accepted practice for inspection of aerospace composites using flash thermography.

In considering the progression of thermographic NDT to its current state, it is important to realize that well into the late 1980's, thermographic NDT was dependent on infrared cameras with (by contemporary standards) poor sensitivity, low pixel densities, frame rates and dynamic ranges, and that analysis was based almost entirely on digitized analog video data. Since then, thermographic NDT has benefited tremendously from subsequent developments in infrared camera technology, as well as the remarkable advancements in personal computers and the capacity for real-time data transfer between the camera and PC. However, these improvements in the underlying technologies only tell part of the story, as they served to enable a better understanding of fundamental thermal interactions by providing more accurate experimental data and facilitating advanced modeling capabilities, leading to the development of the physics-based signal processing techniques that are used in modern systems. The net result of this evolution is that the modern thermographic NDT is capable of providing quantitative, unambiguous results that rival, and in some cases, outperform more mature NDT technologies such as ultrasound or radiography. The range of problems that thermography can address has grown to include detection and measurements of small and subtle subsurface features that would be undetectable using conventional approaches to thermography.

At present, the most widely-implemented form of active thermography uses light to heat the sample surface. The precise configuration of the method is largely a matter of geography. The lock-in technique, in which the applied light is modulated over several on-off cycles, is popular in Europe, while the flash technique, in which a single pulse from a flash lamp array heats the surface, is widely used in the United States. Although much is made of the differences between these techniques, fundamentally, they rely on the same thermophysical phenomenon, namely, the deterministic nature of heat as it diffuses from the surface of a sample to its interior. The essential differences in these methods lies in the mathematical approaches they use to distinguish between normal and anomalous sample behavior. In most cases, the choice between the two approaches comes down to practical considerations, e.g. time, space or cost constraints.

In this presentation, we will review recent developments in flash thermography. In particular, we will describe the Thermographic Signal Reconstruction method, and the quantitative analysis of thermographic derivatives which it facilitates. In doing so, we will trace the evolution of flash thermography from its origins as a qualitative NDT technique based on visual image interpretation to a highly sensitive quantitative method in which a significant amount of information can be extracted from a single pixel time history. 
http://dx.doi.org/10.21611/qirt.2008.01_03_03 